\title{
External high-quality-factor resonator tunes up nuclear magnetic resonance
}

\author{
Martin Suefke ${ }^{1^{*}}$, Alexander Liebisch ${ }^{2}$, Bernhard Blümich $^{2}$ and Stephan Appelt ${ }^{2,3}$ \\ ${ }^{1}$ Institute of Energy and Climate Research - Fundamental Electrochemistry (IEK-9), \\ Forschungszentrum Jülich GmbH, D-52425 Jülich, Germany. \\ ${ }^{2}$ Institut für Technische Chemie und Makromolekulare Chemie (ITMC), \\ RWTH Aachen University, D-52056 Aachen, Germany. \\ ${ }^{3}$ Central Institute for Engineering, Electronics and Analytics - Electronic Systems (ZEA-2), \\ Forschungszentrum Jülich GmbH, D-52425 Jülich, Germany \\ *e-mail: martin.suefke@rwth-aachen.de
}

The development of powerful sensors for the detection of weak electromagnetic fields is crucial for many spectroscopic applications, in particular, for nuclear magnetic resonance (NMR). Here we present a comprehensive, new theoretical model for boosting the signalto-noise ratio, validated by liquid-state ${ }^{1} \mathrm{H},{ }^{129} \mathrm{Xe}$, and ${ }^{6} \mathrm{Li} \mathrm{NMR}$ experiments at low frequencies $(20-500 \mathrm{kHz})$, using an external resonator with a high quality factor combined with a low-quality-factor input coil. In addition to a very high signal-to-noise ratio, this external high quality-factor enhanced NMR exhibits striking features such as a large flexibility with respect to input coil parameters, and a square-root dependence on the sample volume, and signifies an important step towards compact NMR spectroscopy at low frequencies with small and large coils.

Nuclear magnetic resonance spectroscopy preferably operates at high magnetic fields (10-24 T) to benefit from high sensitivity and high chemical shift dispersion. The signal is detected by nuclear induction in coils, which are part of a resonant circuit ${ }^{1-3}$. NMR operating at high frequencies $(\sim 500 \mathrm{MHz})$ is particularly in demand for microcoil-based spectroscopy with sample volumes smaller than $1 \mu \mathrm{L}$ (ref. 4). Microcoils, with their small inductance $(L<1 \mathrm{nH})$, cannot readily be tuned to lower resonance frequencies $(<1 \mathrm{MHz})$ owing to the large capacity $(C>1 \mathrm{mF})$ necessary to fulfil the resonance condition $\omega=(L C)^{-1 / 2}$. Sillerud et al. ${ }^{5}$ were the first to address this problem by adding an external inductor to a microcoil for NMR detection at $40 \mathrm{MHz}$. Coffey et al. ${ }^{6}$ reported a very weak frequency dependence of the signal-to-noise ratio $(S N R)$ when comparing hyperpolarized NMR/MRI at $0.047 \mathrm{~T}$ and $4.7 \mathrm{~T}$ (keeping the polarization constant), with a possibly higher $S N R$ of hyperpolarized low-field NMR/MRI.

So far, NMR spectroscopy with microcoil detection and a large $S N R$ have not be realized at very low Larmor frequencies $\omega_{0} / 2 \pi=\gamma B_{0}=1-1000 \mathrm{kHz}$, where $\gamma$ is the gyromagnetic ratio of the nuclear spin species and $B_{0}$ is the strength of the static magnetic field. Instead, other unconventional schemes for NMR detection are being explored at low frequencies. Such lowfrequency NMR field sensors are superconducting quantum interference devices (SQUIDs, refs 7,8 ) and atomic magnetometers ${ }^{9,10}$ with sensitivities better than $1 \mathrm{fT} / \mathrm{Hz}^{1 / 2}$. Moreover, a single nitrogen vacancy $(\mathrm{NV})$ centre in diamond can detect 100 out of $10^{4}$ proton spins, which are 
statistically polarized ${ }^{11}$, and even a single ${ }^{13} \mathrm{C} \operatorname{spin}^{12}$. However, these sensors have several disadvantages: SQUIDs need liquid nitrogen and helium, and atomic magnetometers operate with lasers at elevated temperatures $\left(100-200^{\circ} \mathrm{C}\right)$. Both sensors require magnetic shields for sensitive detection. Spin-exchange relaxation-free (SERF)-based atomic magnetometers have their highest sensitivity at zero or at ultra-low field $\left(<<10^{-4} \mathrm{~T}\right)$, where the chemical shift information is absent for most nuclear species ${ }^{13-15}$. For NV centres, a large number of measurements (about $10^{6}$ ) is required to generate one complete NMR spectrum, and the linewidth of the signal is broad $(>10 \mathrm{kHz})$ owing to diffusion on the nanoscale, measuring protocols, and instrumental filter functions ${ }^{11}$.

To substantially enhance the $S N R$ of NMR spectroscopy ${ }^{3}$ in the low frequency regime $(1 \mathrm{kHz}-10 \mathrm{MHz})$, this paper introduces the method of "external high-quality-factor enhanced NMR" (EHQE-NMR, Fig. 1a). The basic principle of EHQE-NMR is to improve the $Q$-factordependent enhancement of the NMR signal ${ }^{1,3}$ by adding an external resonator with a high $Q$ value. This resonator consists of a coil distant from the sample with $Q_{\mathrm{E}}=\omega L_{\mathrm{E}} / R_{\mathrm{E}}>>100$ ( $L_{\mathrm{E}}$ : inductance, $R_{\mathrm{E}}$ : resistance) and a tuning capacitor $C\left(Q_{\mathrm{C}} \sim 10000\right)$, and is connected through a low-loss transfer line to the input coil with $Q=\omega L / R$ ( $L$ : inductance, $R$ : resistance). Together, these components form a resonance circuit which is characterized by a total quality factor $Q_{\mathrm{tot}}=\omega L_{\mathrm{tot}} / R_{\mathrm{tot}}$, where $L_{\mathrm{tot}}=L_{\mathrm{E}}+L$ and $R_{\mathrm{tot}}=R+R_{\mathrm{E}}$ are the total inductance and resistance at the operating frequency, respectively. The key feature of EHQE-NMR is the amplification of the NMR signal by a factor, given by the reduced quality factor $Q_{\text {red }}=Q_{\mathrm{E}} /\left(1+R / R_{\mathrm{E}}\right)>>100$. This is much larger than the $Q$-factor of a conventional NMR resonance circuit $(Q<30)$.

The key physics of EHQE-NMR is reported in this work and detailed in the Supplementary Information. By assuming a constant initial nuclear spin polarization $P$ independent of frequency $\omega$ and Johnson noise from the resonant circuit $N=\left(4 k_{\mathrm{B}} T R_{\text {tot }} Q_{\text {tot }}^{2} \Delta v\right)^{1 / 2}$, where $\Delta v=\omega /\left(2 \pi Q_{\text {tot }}\right)$ is the detection bandwidth, we obtain:

$$
S N R=\frac{\pi^{3 / 2}}{\sqrt{k_{\mathrm{B}} T}} \frac{Q_{\mathrm{E}}}{\left(1+R / R_{\mathrm{E}}\right)} \frac{\mu_{0} k_{0} M_{0} D_{\mathrm{i}}^{2}}{\sqrt{L+L_{\mathrm{E}}}}\left(n-\frac{n^{2}}{4 W^{2}}+\frac{n^{3}}{24 W^{4}}\right)
$$

where $\mu_{0}$ is the vacuum permeability, $k_{\mathbf{B}}$ the Boltzmann constant, and $T$ the absolute temperature. Moreover, $M_{0}=0.5 \gamma h n_{s} V P$ is the magnetization of the sample, which is proportional to $\gamma$, Planck's constant $h$, spin number density $n_{\mathrm{s}}$, the sample volume $V$ and nuclear spin polarization $P$. The term $k_{0}$ is a factor accounting for the field inhomogeneity of the input $\mathrm{coil}^{3,4}$. The input coil parameters in equation (1) for a thick cylindrical coil are the inner diameter $D_{\mathrm{i}}$ and the height $H$. For simplicity, $D_{\mathrm{i}}=H$ is assumed. The total number of turns $n$ and the number of turns per layer $W=H / d$ ( $d$ : wire diameter) implicitly yield the outer diameter $D_{\text {a }}$ of a multilayer coil. The three terms proportional to $n, n^{2}$, and $n^{3}$ on the right-hand side of equation (1) describe the sensitivity $B_{1} / i$ (field per unit current) of a multilayer cylindrical coil. The quantities with $n^{2}$ and $n^{3}$ describe the deviation from the known sensitivity ${ }^{3}$ $B_{1} / \mathrm{I}=\mu_{0} n /\left(2^{1 / 2} D_{\mathrm{i}}\right)$ of a thin cylindrical coil. On the right-hand side of equation (1), the reduced quality factor $Q_{\text {red }}=Q_{\mathrm{E}} /\left(1+R / R_{\mathrm{E}}\right)$ appears. This represents the enhancement factor of the NMR 
signal. Experimental and theoretical studies show that large values $Q_{\text {red }} \gg 100$ can be obtained if an external coil with a core of low-loss ferrite material or a superconducting coil is used.

Equation (1) and a model for the parameters $R$ and $L$ represent the core of EHQE-NMR theory (Supplementary Sections III, IV). The key features result from the analysis of the $S N R$ as a function of $n$ and the sample volume $V=\pi D_{\mathrm{i}}^{3} / 4$. The dependence of $S N R$ on $n$ can be described by the multiplication of a linear function with a hyperbolic function of $n$. The linear function can be found in the right-hand bracket of equation (1) (by ignoring small nonlinear terms in $n$ ), and the hyperbolic decay originates from the term $1 /\left(1+R / R_{\mathrm{E}}\right)$, where $R$ is proportional to $n$ to the first order. The resulting curve has a maximum $S N R_{\max }$ at $n_{\max }=\left(3 L_{\mathrm{E}} R_{\mathrm{E}} / 2 \pi \mu_{0} \rho W^{2}\right)^{1 / 3}$, where $\rho$ denotes the specific electrical resistance of the copper wire (Fig. 3). EHQE theory also predicts the maximum values of $S N R_{\max }(\omega)$ as a function of frequency (see below). In addition, EHQE-NMR is highly flexible with respect to the size and shape of the input coil. To show this, three different kinds of input coils were used (Fig.1b): a standard coil, a microcoil, and a surface coil. For a given frequency, each coil operates with the same external resonator and NMR hardware. For example, the ${ }^{1} \mathrm{H}$ NMR free induction decay (FID) from $V=0.9 \mu \mathrm{L}$ benzene which transforms into the spectrum shown in the inset, was detected with a microcoil $\left(D_{\mathrm{i}} \sim 1.3 \mathrm{~mm}\right)$ at $500 \mathrm{kHz}$ in a single scan (Fig. 1c).

Furthermore, with EHQE-NMR high-resolution and chemical shift-resolved spectra for highgamma $\left({ }^{1} \mathrm{H}\right)$ as well as for low-gamma nuclear spins $\left({ }^{6} \mathrm{Li},{ }^{129} \mathrm{Xe}\right)$ can be measured (Fig. $\left.2 \mathrm{a}-\mathrm{d}\right)$. For the FIDs in Fig. $2 \mathrm{a}-\mathrm{c}$, the ${ }^{1} \mathrm{H}$ and ${ }^{6} \mathrm{Li}$ spins were first prepolarized at $B_{\mathrm{p}}=2 \mathrm{~T}$, then transported into the input coil $\left(D_{\mathrm{i}}=1 \mathrm{~cm}, n=80, W=63\right)$, and, after $\pi / 2$ pulse excitation, the NMR signal was recorded at $500 \mathrm{kHz}\left({ }^{1} \mathrm{H}\right)$ or at $83 \mathrm{kHz}\left({ }^{6} \mathrm{Li}\right)$. A large $S N R=172$ is obtained at $500 \mathrm{kHz}$ with an external coil having a ferrite core $\left(Q_{\mathrm{E}}=218\right)$ and for a ${ }^{1} \mathrm{H}$ prepolarization of $P=1.5 \times 10^{-6}$ (Fig. 2a).

Even at very low frequency, the ${ }^{1} \mathrm{H}$ chemical shift can be resolved as demonstrated by the NMR spectrum of $50 \mu \mathrm{L}$ acetic acid measured with a small input coil $\left(D_{\mathrm{i}}=5 \mathrm{~mm}\right.$, Fig. $\left.2 \mathrm{~b}\right)$. The leftand right-hand lines in the spectrum (inset) can be assigned to the $\mathrm{CH}_{3}$ and the $\mathrm{COOH}$ groups of acetic acid, respectively. The frequency difference of $5 \mathrm{~Hz}$ corresponds to a chemical shift difference of $10 \mathrm{ppm}$ between both chemical groups, thus agreeing perfectly with high-field measurements. The measurement of rare nuclei at low frequencies is difficult owing to the small numbers of spins and the low spin polarization. Nevertheless, ${ }^{6} \mathrm{LiCl}$ dissolved in $\mathrm{H}_{2} \mathrm{O}$ ( $V=450 \mu \mathrm{L}, P \sim 10^{-6}$ ) can be detected with a single scan at $83 \mathrm{kHz}$ (Fig. 2c).

With the present setup, the limit of detection (LOD) for a saturated ${ }^{6} \mathrm{LiCl}$ solution is about 10 $\mu \mathrm{L}$. In the future, the LOD may be extended even further so that ${ }^{6} \mathrm{Li}^{+}$ions in batteries or in neurons may become measurable using external resonators with $Q_{\text {red }} \gg 300$ and better hyperpolarization technologies ${ }^{16-20}$.

With the EHQE technology, NMR signals of hyperpolarized ${ }^{129} \mathrm{Xe}$ exposed to normal atmospheric conditions $\left(21 \% \mathrm{O}_{2}\right)$ can be detected on a surface (Fig. 2d). The ${ }^{129} \mathrm{Xe}$ FID (inset) is measured at $166 \mathrm{kHz}$ using a surface coil (Fig. 1b, right). A continuous flow of hyperpolarized $\mathrm{Xe}$ gas was released from a nozzle with a microchannel into the air, from where it migrated into the liquid toluene surface (Fig.1b, right). The ${ }^{129} \mathrm{Xe}$ NMR spectrum (Fig. 2d) shows a small 
broad line (Xe gas) which is separated from the large narrow line (Xe in toluene) by $33 \mathrm{~Hz}$. This corresponds to a chemical shift difference of $185 \mathrm{ppm}$ and demonstrates the suitability of continuous-flow hyperpolarized Xe EHQE-NMR for the real-time chemical analysis of surfaces.

The EHQE theory elaborated in the Supplementary Information was investigated by performing various ${ }^{1} \mathrm{H}$ NMR experiments where only one parameter, e.g. the number $n$ of turns in the coil, was changed and all other experimental parameters $\left(P, Q_{\mathrm{E}}, L_{\mathrm{E}}, R_{\mathrm{E}}, V, D_{\mathrm{i}}, d, W\right)$ were kept constant. The five plots in Fig. 3 top and bottom show the measured $S N R$ as a function of $n$ where each plot corresponds to a frequency value $\omega / 2 \pi=500 \mathrm{kHz}, 166 \mathrm{kHz}, 83 \mathrm{kHz}, 41 \mathrm{kHz}$, or $20 \mathrm{kHz}$. The solid lines represent the $S N R$ as derived from equation (1) with the given input parameters. The theoretical prediction is in good agreement with the experimental data. All essential features - namely the initial linear increase in $S N R$, the appearance of a maximum at $n_{\max }=\left(3 L_{\mathrm{E}} R_{\mathrm{E}} / 2 \pi \mu_{0} \rho W^{2}\right)^{1 / 3}$ and the hyperbolic decrease for $n>n_{\max }$ - are found in the experimental results. The measured values for $n_{\max }=90,160$ for $v=500 \mathrm{kHz}$ and $166 \mathrm{kHz}$ agree well with the theoretical $n_{\text {max }}$.

As the experimental data clearly supports the proposed EHQE theory, the theory can be used to simulate complex EHQE-NMR scenarios - for example, the best $S N R$ in a multidimensional parameter space (Supplementary Section VII). Regarding the current development of miniaturized NMR spectrometers, two essential building blocks appear to be crucial: a small well-shimmed permanent magnet ${ }^{21}$ or electromagnet ${ }^{22}$ and a sensitive detector for small samples (for example a microcoil). This realization prompted an experimental study into how the SNR of EHQE-NMR depends on $\omega$ and $V$; because small magnets with high homogeneity are associated with weak magnetic fields and low $\omega$, and the vanishing signal sets a lower boundary on $V$ at $S N R \sim 1$.

Thus, in Fig. 4a, the $S N R_{\max }$ as determined from the $S N R$ maxima in Fig. 3 are plotted as a function of $\omega$. The solid line corresponds to $S N R_{\max }$ according to EHQE theory, including the skin and proximity effects, and assuming a constant $Q_{\mathrm{E}}=314$ and $d=0.12 \mathrm{~mm}$. The experimental results are in close agreement with the predicted values, showing the weak dependence of the $S N R_{\max }(\omega)$ in the frequency range examined. Details addressing a broader frequency range $(1 \mathrm{kHz}-10 \mathrm{MHz})$ and more parameters are discussed in Supplementary Section VII. Our results imply that NMR signals can be acquired in single scans using EHQE in the frequency range from $1 \mathrm{kHz}-10 \mathrm{MHz}$ for a large number of high- and low- gamma spins and for a wide range of sample volumes. The corresponding magnetic field $B_{0}$ can be provided by permanent magnets, electromagnets ${ }^{22}$ or even the Earth's magnetic field ${ }^{23}$.

Further experimental evidence in support of the EHQE theory is provided by the $S N R$ as a function of $V$ for two different experimental conditions (Fig. 4b). In a first scenario, $V$ was decreased inside a fixed input coil $\left(D_{\mathrm{i}}=H=1 \mathrm{~cm}\right)$; the measured points (open squares) agree well with $S N R \sim V$ (solid line), as both standard and EHQE-NMR theory claim. In the second scenario, the size of the input coil was reduced in proportion to the sample volume $V$; the measured $S N R$ (open circles) scales with $S N R \sim V^{1 / 2}$ (dashed line). For $N_{s}=10^{22}$ proton spins $\left(V \sim(0.6 \mathrm{~cm})^{3}\right.$ benzene) and $P=10^{-6}$, when using an optimized wire diameter, our best experiments so far show $S N R_{\max } \sim 1000$. As $S N R \sim V^{1 / 2} \sim N_{s}^{1 / 2}$, the LOD for a single scan is 
$10^{16}$ proton spins, corresponding to $V \sim(65 \mu \mathrm{m})^{3}$. For $N_{s}=10^{16}$ spins at $P=10^{-6}$ we have $10^{10}$ spins thermally polarized, in contrast to $N_{s}^{1 / 2}=10^{8}$ spins which are polarized statistically. Statistical polarization is a form of spin noise ${ }^{24}$ where spontaneously created transverse magnetization components $\sim N_{s}^{1 / 2}$ oscillate at the Larmor frequency around the $B_{0}$ field ${ }^{11,25}$. If the present $S N R_{\max } \sim 1000$ is increased by more than a factor of 100 , this would give LOD $\sim 10^{12}$ spins $\left(V \sim(3 \mu \mathrm{m})^{3}\right)$ and the number of statistically polarized spins is equal to the number of thermally polarized spins (both $10^{6}$ ). Below this crossover point at $N_{s}<10^{12}$ spins, a high- $Q$ EHQE microresonator with $Q_{\mathrm{E}}>10^{5}$ should be able to measure NMR spectra at 300 $\mathrm{K}$ without any prepolarization.

The $S N R$ can be calculated as a function of $n$ for three external coils (Fig. 4c), all with $L_{\mathrm{E}}=0.11 \mathrm{mH}$ but with three different values of $Q_{\mathrm{E}}=218$ (ferrite, as in Fig. 3, circles), $Q_{\mathrm{E}}=1300$ (coil at $T=77 \mathrm{~K}$ ), and $Q_{\mathrm{E}}=$ infinity (superconductive coil). The improvement in the simulated $S N R$ from the ferrite to the superconducting coil is about two orders of magnitude. The maximum $S N R$ for the superconducting coil is shifted to $n=1$. If the $S N R$ is further increased using a smaller detection bandwidth, then EHQE-NMR should be feasible in the $\mu \mathrm{m}$ regime based on statistical polarization of $N_{s} \sim 10^{12}$ spins.

In conclusion, this work reports the power of ultra-sensitive inductive detection of signals at low frequencies by EHQE-NMR. This technique is based on high- $Q$ external resonators, and the $S N R$ scales with the sample volume as $V^{1 / 2}$. There are basically no constraints on the geometry of the input coil. This, in particular, allows the use of microcoils as well as the use of surface- and large volume coils. These key features enable amongst others the development of miniaturized NMR sensors for high-throughput analysis and personal health care ${ }^{26}$. As EHQENMR works best in the low-frequency regime $(1 \mathrm{kHz}-10 \mathrm{MHz})$, it is also suitable for the sensitive detection of low-gamma spins, such as ${ }^{103} \mathrm{Rh},{ }^{107} \mathrm{Ag},{ }^{183} \mathrm{~W}$ and ${ }^{39} \mathrm{~K}$ at fields up to several tesla. This could have important implications for the real-time monitoring of catalytic processes ( $\mathrm{Rh}, \mathrm{Ag}, \mathrm{W}$ ). Improvement of in vivo ${ }^{39} \mathrm{~K}$ and ${ }^{23} \mathrm{Na} \mathrm{MRI}$ at high fields ${ }^{27}$ is possible by performing EHQE NMR detection at fields of $0.5-3 \mathrm{~T}$ (Larmor frequency $<10 \mathrm{MHz}$ ), and by using pure phase encoding imaging sequences, in which the signals are measured at a narrow bandwidth in the absence of magnetic field gradients.

Last, but not least, the EHQE method is not restricted just to NMR in material and medical sciences, but can be used to detect all kinds of weak electromagnetic fields at low frequency. EHQE resonators could be useful for detecting magnetic nanoparticles (MPI; ref 28) or the signal of an oscillating electron spin ensemble (ESR) at low frequencies. One specific example is a miniaturized magnetic field sensor based on stroboscopic optical excitation of alkaline vapour $^{29}$, which uses a light-emitting diode and an EHQE resonator instead of a pump and detection laser. Optimized EHQE resonance circuits could also be used instead of SQUIDs for the implementation of a two nuclei co-magnetometer ${ }^{30,31}$ for the search of parity- and timeviolating processes or axion-like particles ${ }^{32}$. The ability to detect small volumes with EQHE resonance circuits could be interesting for exploring quantum electrodynamics (QED) effects in NMR such as the virtual photon exchange between spins and the surrounding detector coil ${ }^{33}$. 


\section{Methods}

\section{Sample preparation:}

A defined amount of liquid benzene $(V=0.7-500 \mu \mathrm{L})$ was filled into several cylindrical glass tubes with different wall thicknesses and inner diameters (1-8 mm). ${ }^{6} \mathrm{LiCl}(98 \%$ enriched) was dissolved in water and $500 \mu \mathrm{L}$ of a $72 \%$-saturated solution was filled into a cylindrical glass tube with an outer diameter of $1 \mathrm{~cm}$ and an inner diameter of $8 \mathrm{~mm}\left(D_{\mathrm{i}}=1 \mathrm{~cm}, V=500 \mu \mathrm{L}\right)$.

\section{EHQE-NMR and hyperpolarization setup:}

A $2 \mathrm{~T}$ Halbach magnet ${ }^{34}$ was used for prepolarization ${ }^{23}$ of ${ }^{1} \mathrm{H}$ and ${ }^{6} \mathrm{Li}$ spins. A continuous flow of hyperpolarized $\mathrm{Xe}$ gas $\left(4 \% \mathrm{Xe}, 10 \% \mathrm{~N}_{2}\right.$, remainder $\mathrm{He}, p=2$ bar) with a polarization $P_{\mathrm{Xe}} \sim 0.3$ was produced using a $\mathrm{Rb}-\mathrm{Xe}$ flow polarizer ${ }^{35}$. The $\mathrm{Xe}$ gas mixture was transported from a plastic tube to a glass nozzle with a diameter of $\sim 70 \mu \mathrm{m}$. A gas flow of $300 \mathrm{~cm}^{3} / \mathrm{min}$ was released into ambient air at a pressure of 1 bar. The hyperpolarized Xe atoms diffused from the gas phase into the liquid sample, located in the centre of the $B_{0}$ field magnet. Five external coils with ferrite cores were used for EHQE-NMR detection at frequencies of 20,41, 83, 166, and $500 \mathrm{kHz}$, respectively. Their measured Q-factors were $Q_{\mathrm{E}}=250,380,369,280$ and 218, respectively. The ferrite coil was placed inside a double $\mathrm{Mu}$-metal shield to prevent magnetic saturation due to stray magnetic fields that would destroy the large Q-factor enhancement of the ferrite coil. As the Mu-metal shield itself is magnetic, it must be far enough away $(\sim 40 \mathrm{~cm})$ from the centre of the $B_{0}$ field coil so that the magnetic field homogeneity in the centre $\left(\sim 1 \mathrm{ppm} / \mathrm{cm}^{3}\right)$ is not destroyed. The ferrite coil is electrically connected to the input coil by means of a transfer line, which is made of thick copper litz wire with very low a.c. resistance.

\section{Measurements:}

The nuclear spins ${ }^{1} \mathrm{H}$ of benzene $\left(T_{1}=3.7 \mathrm{~s}\right)$ and ${ }^{6} \mathrm{Li}$ in water $\left(T_{1} \sim 1000 \mathrm{~s}\right)$ were first prepolarized using a $2 \mathrm{~T}$ Halbach magnet, thereby attaining a nuclear polarization of $P_{\mathrm{H}}=6 \cdot 10^{-6}$ and $P_{6 \mathrm{Li}}=8.8 \cdot 10^{-7}$. They were then placed by hand into the input coil. After $5 \mathrm{~s}$, the longitudinal ${ }^{6} \mathrm{Li}$ magnetization was still the same, while the ${ }^{1} \mathrm{H}$ magnetization decayed to $P_{\mathrm{H}}=1.55 \cdot 10^{-6}$. Transverse magnetization was generated by a $90^{\circ}$ radiofrequency pulse from a saddle coil. Subsequently, the FID was measured by the EHQE resonator, which was then further amplified by a preamplifier and a lock-in amplifier (bandwidth $=\Delta v$ ) before being recorded. Two series of input coil modules were used for $S N R$ studies: The first series consisted of twelve coils, all of which had a $D_{\mathrm{i}}=H=1 \mathrm{~cm}$ and were wound with enamelled copper wire ( $d=0.12 \mathrm{~mm}$ ) but with varying $n=26-900$. The second series comprised eight coils with a constant $n=80$, but with decreasing $D_{\mathrm{i}}$ and $d$ from $1 \mathrm{~cm}$ to $1.3 \mathrm{~mm}$ and from $0.12 \mathrm{~mm}$ to 0.05 $\mathrm{mm}$, respectively. 


\section{References}

1. Abragam, A. The Principles of Nuclear Magnetism (Clarendon, 1961).

2. Ernst, R. R., Bodenhausen, G. \& Wokaun, A. Principles of Nuclear Magnetic Resonance in One and Two Dimensions (Clarendon, 1987).

3. Hoult, D. I., Richards, R. E. The signal-to noise ratio of nuclear resonance experiment. Journal of Magnetic Resonance 24, 71-85 (1976).

4. Webb, A. G. Radiofrequency microcoils in magnetic resonance. Progress in Nuclear Magnetic Resonance Spectroscopy 31, 1-42 (1997).

5. Sillerud, L. O. et al. ${ }^{1} \mathrm{H}$ NMR Detection of superparamagnetic nanoparticles using a microcoil and novel tuning circuit. Journal of Magnetic Resonance 181, 181-190 (2006).

6. Coffey, A. M., Truong, M. L., Chekmenev, E. Y. Low-field MRI can be more sensitive than high-field MRI. Journal of Magnetic Resonance 237, 169-174 (2013).

7. Greenberg, Y. S. Application of superconducting quantum interference devices to nuclear magnetic resonance. Rev. Mod. Phys.70, 175-222 (1998).

8. McDermott, R. et al. Liquid-State NMR and Scalar Couplings in Microtesla Magnetic Fields. Science 295, 2247-2249 (2002).

9. Kominis, I. K., Kornack, T. W., Allred, J. C., Romalis, M. V. A subfemtotesla multichannel atomic magnetometer. Nature 422, 596-599 (2003).

10. Sheng, D., Li, S., Dural, N., Romalis, M. V. Subfemtotesla Scalar Atomic Magnetometry Using Multipass Cells. Phys. Rev. Lett. 110, 160802 (2013).

11. Staudacher, T., et al. Nuclear Magnetic Resonance Spectroscopy on a (5-Nanometer) ${ }^{3}$ Sample Volume. Science 339, 561-563 (2013).

12. Jelezko, F., et al. Observation of coherent oscillation of a single nuclear spin and realization of a two-qubit conditional quantum gate. Phys. Rev. Lett. 93, 130501 (2004).

13. Appelt, S., Häsing, F. W., Kühn, H., Perlo, J. and Blümich, B. Mobile High Resolution Xenon Nuclear Magnetic Resonance Spectroscopy in the Earth's Magnetic Field. Phys. Rev. Lett. 94, 197602 (2005).

14. Theis, T. et al. A. Parahydrogen-enhanced zero-field nuclear magnetic resonance. Nat. Phys. 7, 571-575 (2011).

15. Ledbetter, M. P. et al. Near-Zero Field Nuclear Magnetic Resonance. Phys. Rev. Lett. 107, 107601 (2011).

16. Happer, W. Optical Pumping. Rev. Mod. Phys. 44, 169-249 (1972).

17. Bouchiat, M. A., Carver, R. R. \& Varnum, C. M. Nuclear Polarisation in $\mathrm{He}^{3}$ Gas Induced by Optical Pumping and Dipolar Exchange. Phys. Rev. Lett. 5, 373-375 (1960).

18. Carver T. R. and Slichter C. P. Experimental Verification of the Overhauser Nuclear Polarization Effect. Phys. Rev. 102, 975-981 (1956). 
19. Bowers, C. R. \& Weitekamp, D. P. Transformation of symmetrization order to nuclearspin magnetisation by chemical reaction and nuclear magnetic resonance. Phys. Rev. Lett. 57, 2645-2648 (1986).

20. Adams R. W. et al. Reversible interactions with para-hydrogen enhance NMR sensitivity by polarization transfer. Science 323, 1708-1711 (2009).

21. Perlo, J. et al. High-Resolution NMR Spectroscopy with a Portable Single-Sided Sensor. Science 308, 1279 (2005).

22. Blümich, B., Casanova, F., Appelt, S. NMR at low magnetic fields. Chemical Physics Letters 477, 231-240, (2009).

23. Appelt, S., Kühn, H., Häsing, F.W. Blümich, B. Chemical analysis by ultrahigh-resolution nuclear magnetic resonance in the Earth's magnetic field. Nat. Phys. 2, 105-109 (2006).

24. Sleator, T., and Hahn, E. L., and Hilbert, C. and Clarke, J. Nuclear Spin Noise. Phys. Rev. Lett. 55, 1742-1745 (1985).

25. Mamin, H. J., Budakian, R., Chui, B. W., and Rugar, D. Magnetic resonance force microscopy of nuclear spins: Detection and manipulation of statistical polarization. Phys. Rev. B, 72, 024413 (2005).

26. Sun, N. et al. Small NMR biomolecular sensors. Solid-State Electronics 84, 13-21 (2013).

27. Augath, M., Heiler, P., Kirsch, S., Schad, L. R. In vivo ${ }^{39} \mathrm{~K},{ }^{23} \mathrm{Na}$ and ${ }^{1} \mathrm{H}$ MR imaging using a triple resonant RF coil setup. Journal of Magnetic Resonance 200, 134-136 (2009).

28. Gleich, B., Weizenecker, J. Tomographic imaging using the nonlinear response of magnetic Particles. Nature 435, 1214-1217 (2005).

29. Wäckerle, G., Appelt, S., and Mehring, M. Two-dimensional optical spectroscopy by periodic excitation of sublevel coherence with sub-Doppler resolution. Phys. Rev. A, 43, 242-250 (1991).

30. Appelt, S., Wäckerle, G., Mehring, M. Geometric Effects in NMR Gyroscopes, in High Precision Navigation 95 (Linkwitz, K., Hangleiter, U., eds.), 29-36 (Dümmler Verlag, Bonn, 1995).

31. Staiber H.-D., Appelt, S., Butscher, R., Wäckerle, G., Mehring, M. Realization of a twonuclei NMR gyroscope, in: High Precision Navigation 95 (Linkwitz, K., Hangleiter, U., eds.), 62-69 (Dümmler Verlag, Bonn, 1995).

32. Tullney, K. et al. Constraints on Spin-Dependent Short-Range Interaction between Nucleons, Phys. Rev. Lett. 111, 100801 (2013).

33. Engelke, F. Virtual photons in magnetic resonance. Concepts in Magnetic Resonance 36A, 266-339 (2010).

34. Halbach, K. Design of permanent multipole magnets with oriented rare earth cobalt material. Nuclear Instruments and Methods 169, 1-10 (1980).

35. Driehuys, B. et al. High-volume production of laser-polarised ${ }^{129} \mathrm{Xe}$. Appl. Phys. Lett. 69, 1668-1670 (1996). 


\section{Acknowledgements}

The authors gratefully acknowledge excellent technical assistance and financial support from Prof. S. van Waasen, Prof. R. Eichel, and A. Schwaitzer at Forschungszentrum Jülich GmbH, and P. Schleker at RWTH Aachen University for sample preparation and consistency checks. We also wish to thank W. Zia for stimulating discussions as well as J. Colell, M. Emondts, and S. Glöggler for their help with various details in hardware construction. Furthermore, we would like to thank M. J. Blümich and H. Burlet for editing the manuscript.

\section{Author contributions}

M. S. designed and built the EHQE-NMR probehead, performed experiments, and evaluated experimental results; A. L. built input coil modules, performed experiments, and analysed experimental results; B. B. provided laboratory and experimental facilities and creative input; S. A. supervised the project, designed the EHQE-NMR setup, performed experiments, and developed the theory. S. A., M. S., A. L., and B. B. wrote the paper.

\section{Competing financial interests}

The authors declare that they have no competing financial interests. 


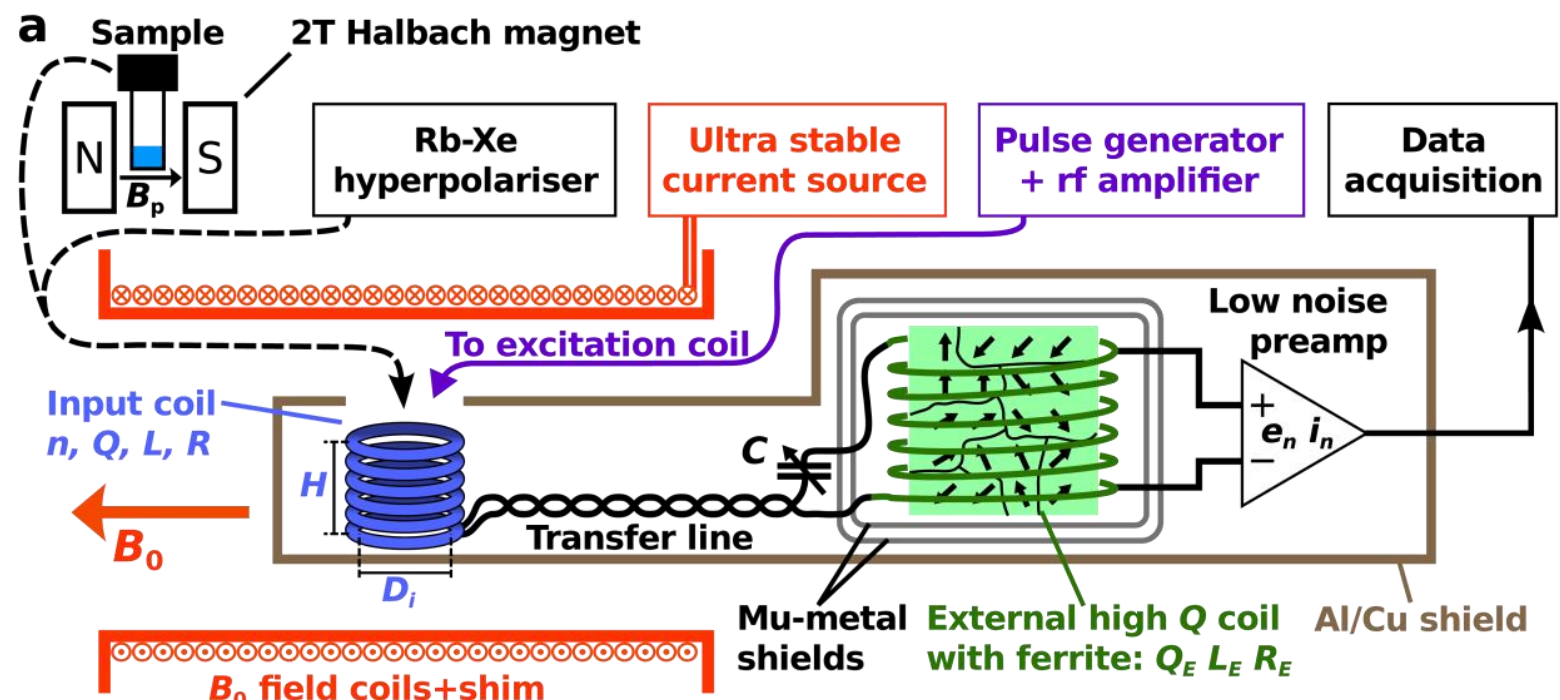

b

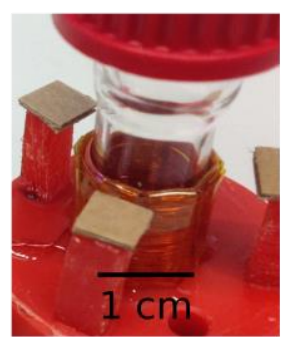

Standard

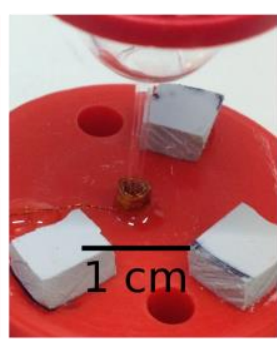

Micro

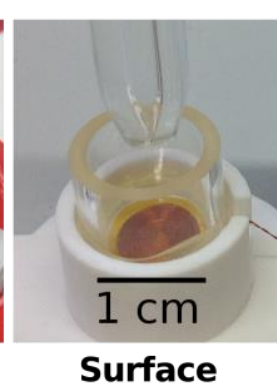

Surface

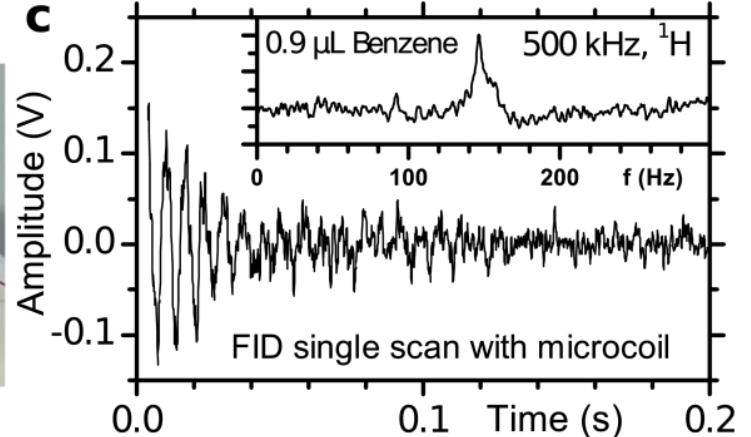

Figure 1 | Experimental setup of the EHQE-NMR spectrometer. a, At the heart of EHQE detection is an external resonator consisting of a tuning capacitor $\left(Q_{\mathrm{C}}>>1000\right)$ and a magnetically shielded coil with a ferrite core $\left(Q_{\mathrm{E}} \gg>100\right)$. A precessing nuclear spin ensemble, prepolarized by either a $2 \mathrm{~T}$ Halbach magnet or a $\mathrm{Rb}$-Xe hyperpolarizer, induces an a.c. voltage in the input coil $(Q, R, L)$ which is transmitted through a transfer line to the external resonator. The NMR signal is amplified by the reduced quality factor $Q_{\text {red }} \gg 100$. For pulse excitation, a saddle coil is used (not shown) whose $B_{1}$ field axis is orthogonal to the sensitive axis of the input coil and to the direction of the $B_{0}$ field. This decoupling is essential for a large $S N R$. $\mathbf{b}$, Three different input coil modules from left to right: cylindrical coil with $D_{\mathrm{i}}=H=1 \mathrm{~cm}$, microcoil with $D_{\mathrm{i}}=H=1.3 \mathrm{~mm}$, and surface coil (100 turns, $8 \mathrm{~mm}$ diameter). The surface coil is located below a sample holder and a nozzle for continuous delivery of hyperpolarized Xe gas. c, Single-scan ${ }^{1} \mathrm{H}$ NMR signal $(500 \mathrm{kHz}, \pi / 2$ pulse $)$ of $0.9 \mu \mathrm{L}$ benzene measured with a microcoil $\left(D_{\mathrm{i}}=1.3 \mathrm{~mm}\right.$, Fig. $\left.1 \mathrm{~b}\right)$. Note the small value of the transverse relaxation time $T_{2} \sim 30$ $\mathrm{ms}$, which is attributed to radiation damping. The inset shows the frequency spectrum. 

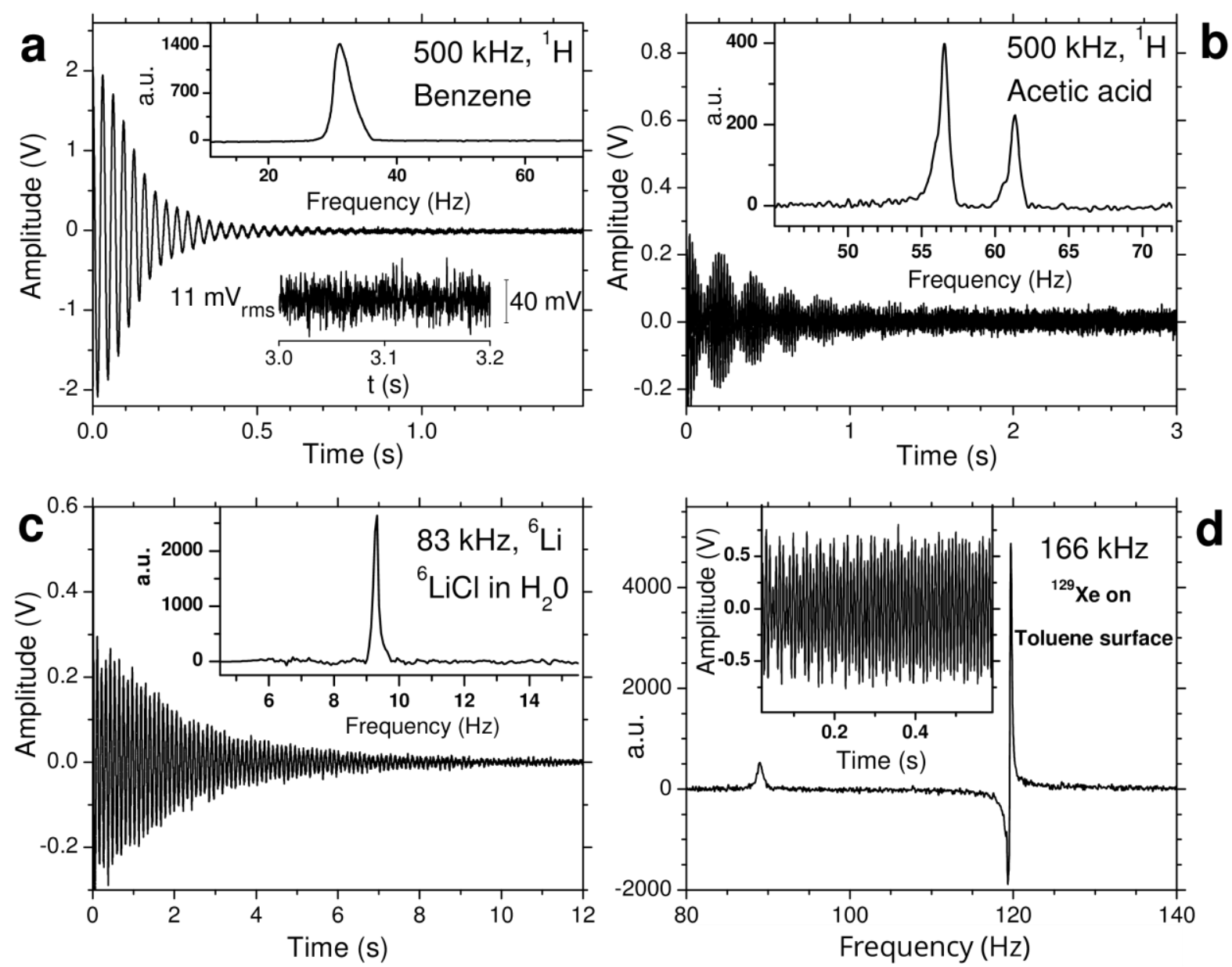

Figure 2 | Single-scan ${ }^{1} \mathrm{H},{ }^{6} \mathrm{Li}$, and ${ }^{129} \mathrm{Xe}$ EHQE-NMR measurements. a, ${ }^{1} \mathrm{H}$-FID and spectrum (upper inset) of $500 \mu \mathrm{L}$ benzene. The $S N R=172$ results from division of the initial amplitude $=2 \mathrm{~V}$ by the r.m.s. noise $=11.7 \mathrm{mV}$ (lower inset). $\mathbf{b},{ }^{1} \mathrm{H}$-FID and spectrum (inset) of $50 \mu \mathrm{L}$ acetic acid $\left(\mathrm{CH}_{3} \mathrm{COOH}\right)$. The results shown in $\mathbf{a}, \mathbf{b}$, are from measurements taken at $500 \mathrm{kHz}\left(B_{0}=117.5 \mathrm{G}\right)$. c, ${ }^{6} \mathrm{Li}$ FID and spectrum (inset) of $450 \mu \mathrm{L}{ }^{6} \mathrm{LiCl}$ dissolved in $\mathrm{H}_{2} \mathrm{O}$ measured at $83 \mathrm{kHz}\left(B_{0}=133 \mathrm{G}\right)$. The spectral linewidth is $0.16 \mathrm{~Hz}$. d, Hyperpolarized ${ }^{129} \mathrm{Xe}$ NMR spectrum of a gas-liquid toluene interface measured at $166 \mathrm{kHz}\left(B_{0}=141 \mathrm{G}\right)$ using a surface coil. The sample investigated consisted of a $3 \mathrm{~mm}$-thick layer of liquid toluene that was placed on a $50 \mu \mathrm{m}$-thick mica plate covering the surface coil. The free Xe gas signal in the FID (inset) dies out after $0.5 \mathrm{~s}$ owing to convection and only the signal of Xe in toluene survives. For a-d, the pulse excitation angle was $\pi / 2$. 


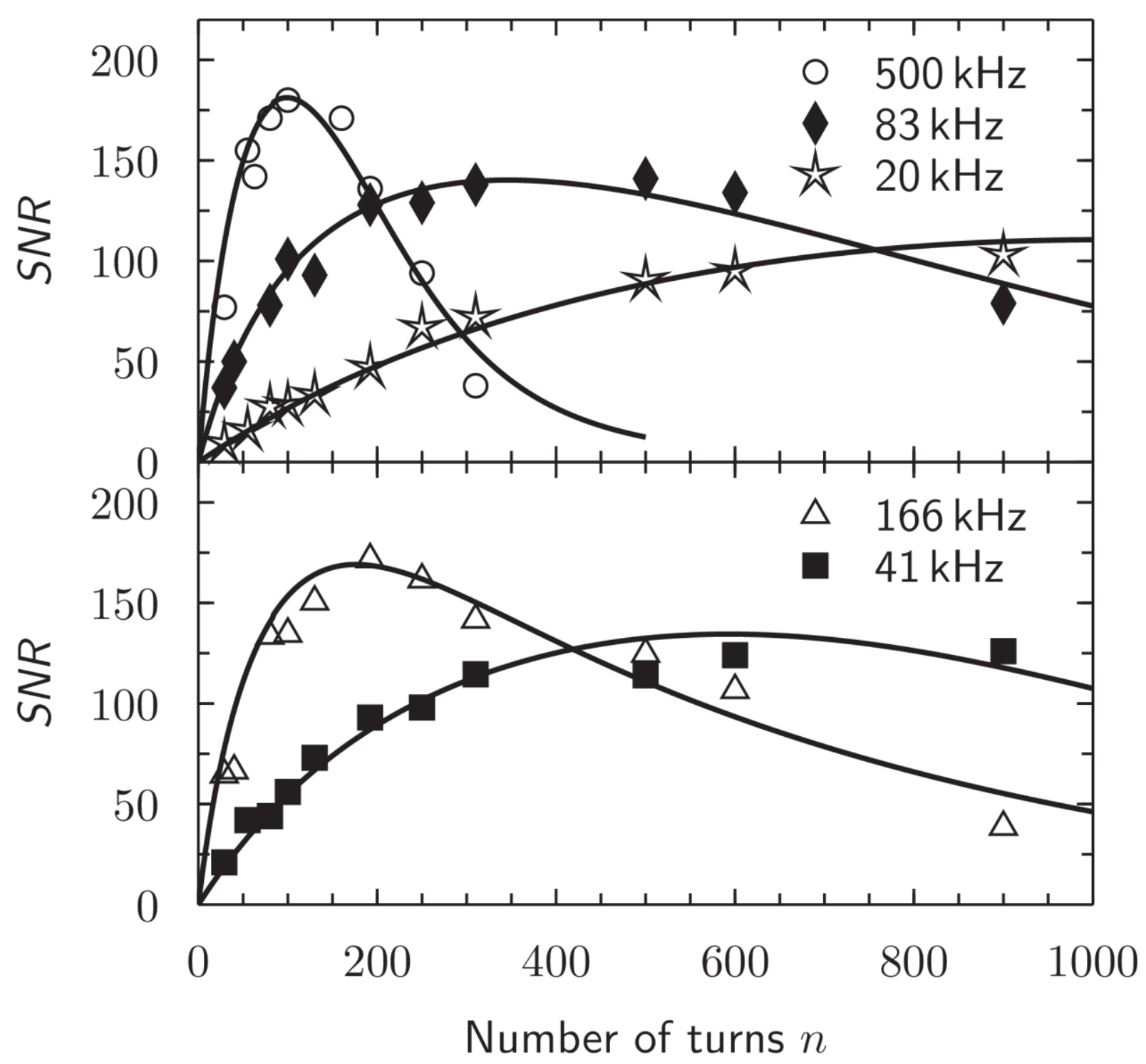

Figure 3 | Experimental confirmation of EHQE-NMR theory. $S N R$ as a function of the number of turns, $n$, of the input coil measured at ${ }^{1} \mathrm{H}$ frequencies $\omega / 2 \pi=500 \mathrm{kHz}, 166 \mathrm{kHz}$, $83 \mathrm{kHz}, 41 \mathrm{kHz}$, and $20 \mathrm{kHz}$ as indicated. For each of the five frequencies, external ferrite coils with different $L_{\mathrm{E}}$ but with similar $Q_{\mathrm{E}} \sim 300$ were used. Solid lines correspond to the EHQE model based on equation (1). Sample: benzene, $V=500 \mu \mathrm{L}, P=1.55 \cdot 10^{-6}$. Input coil parameters: $D_{\mathrm{i}}=H=1 \mathrm{~cm}, W=63, d=0.12 \mathrm{~mm}$. At lower frequencies, the maximal $S N R_{\max }$ shifts to higher values of $n_{\max }$. 

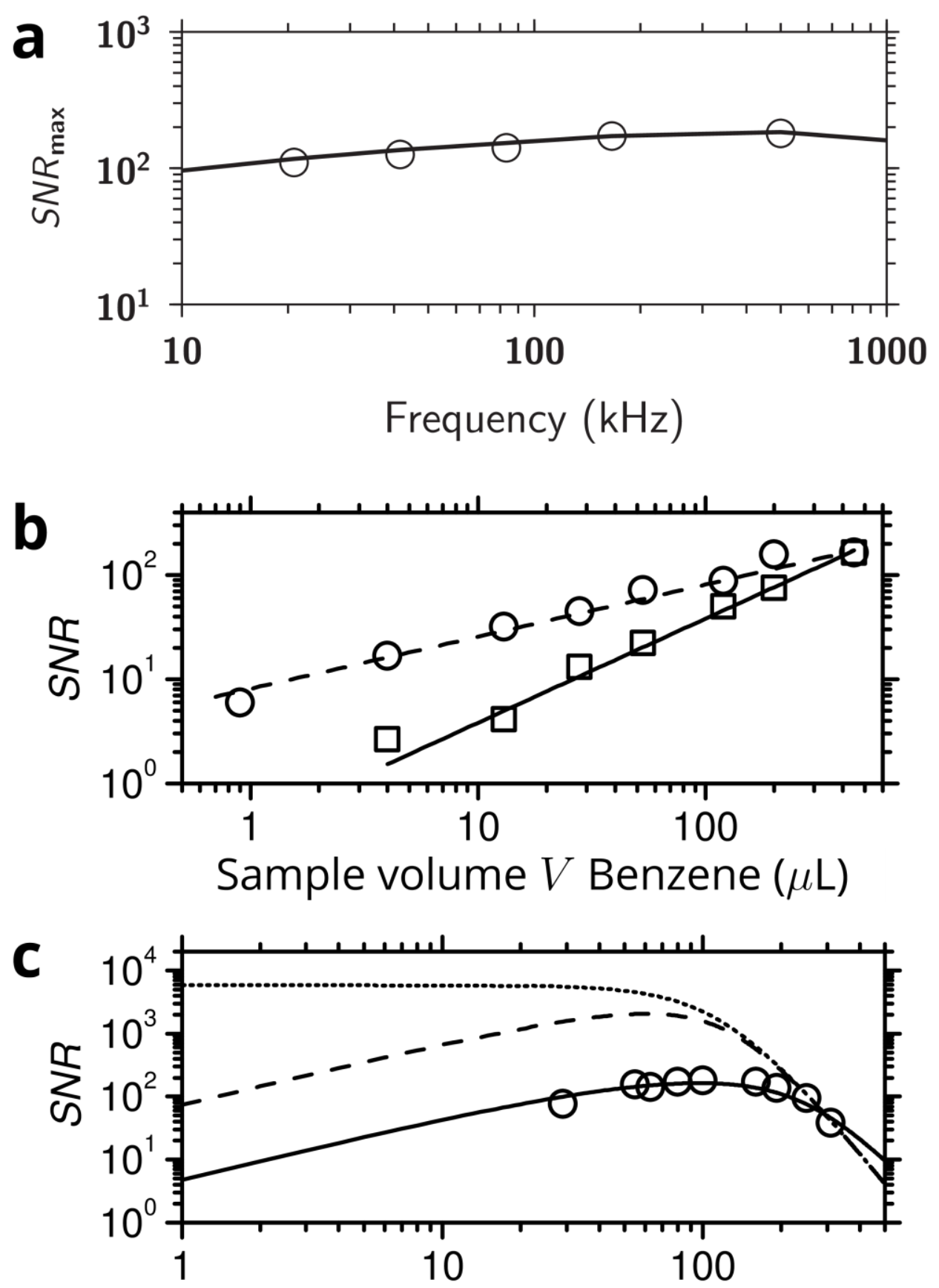

Number of turns $n$

Figure 4 | Key properties of the EHQE method using ${ }^{1} \mathrm{H}-\mathrm{NMR}$ of benzene. a, $S N R_{\max }$ as a function of frequency $\omega / 2 \pi$. The five values (open circles) are taken from the maxima in Fig. 3. Data are in agreement with EHQE theory (solid line). b, SNR as a function of $V$ measured with a fixed input coil and variable $V$ (open squares) or by shrinking the input coil together with $V$ in the same proportion (open circles). Solid lines correspond to $S N R \sim V$ and dashed lines to $S N R \sim V^{1 / 2}$. c, Simulated $S N R$ as a function of $n$ assuming a detection frequency $\omega / 2 \pi=500 \mathrm{kHz}$ and three different external coils: ferrite coil $\left(Q_{\mathrm{E}}=218\right.$, solid line), cylindrical coil at $T=77 \mathrm{~K}$ $\left(Q_{\mathrm{E}}=1300\right.$, dashed line $)$, and superconducting coil $\left(Q_{\mathrm{E}}=\right.$ infinity, dotted line $)$. Input coil parameters: $D_{\mathrm{i}}=H=1 \mathrm{~cm}, d=0.12 \mathrm{~mm}$ (solid line), and $45 \times 0.07 \mathrm{~mm}$ litz wire (dashed, dotted line). Circles indicate measured values. 